\title{
SYNTHESIS, MOLECULAR DOCKING AND ANTITUBERCULAR ACTIVITY OF NEW BI HETEROCYCLIC COMPOUNDS ON BENZIMIDAZOLE MOIETY
}

\author{
Dhanaja Kotte ${ }^{1}$, Kumaraswamy Gullapelli ${ }^{2}$, Ravichandar Maroju ${ }^{2}$ \\ Ramchander Merugu ${ }^{3}$, Brahmeshwari Gavaji ${ }^{1, *}$ \\ ${ }^{1}$ Department of Chemistry, Kakatiya University, 506009, Warangal - India \\ ${ }^{2}$ Department of Chemistry, Mahatma Gandhi Institute of Technology, 500075, Hyderabad -India \\ ${ }^{3}$ Department of Bio-Chemistry, Mahatma Gandhi University, 508254, Nalgonda- India \\ *E-mail: gbrahmeshwari@gmail.com
}

\begin{abstract}
A series of new and efficient Benzo [d] imidazole-2-yl -3, 5 substituted diphenyl -3,3a.5.6 -Tetrahydro 2H-pyrazole thiazole derivatives (4a-j) were synthesized from Schiff base derivatives of thiazolidinediones (3a-j) using hydrazine hydrate. The synthesized compounds were screened for their antitubercular and molecular docking studies. The results of docking studies are supporting antitubercular activity showing high inhibition constant and binding energy. The structures of synthesized compounds were characterized by IR, ${ }^{1} \mathrm{HNMR}$, Mass spectroscopic methods. Keywords: Synthesis, Molecular Docking, Antitubercular Activity, Benzimidazole Derivatives
\end{abstract}

(C) RASĀYAN. All rights reserved

\section{INTRODUCTION}

The quest for synthesis of novel materials for specific applications has become a great challenge for researchers aiming to address urgent real-world demands. ${ }^{1-2}$ To meet this target, significant attention has been paid to design and develop heterocyclic molecules owning desirable properties. ${ }^{3-4}$

Heterocyclic chemistry is highly challenging especially in the growing demand for higher efficiency and eco-friendly synthesis. ${ }^{5-6}$ It always attracts the attention of scientists working in the area of natural products and synthesis of heterocyclic compounds, especially with nitrogen-containing heterocyclic molecules that occupy the key position in the area of drugs and pharmaceuticals. ${ }^{7-9}$ Majority of the pharmaceutical products are heterocyclic molecules that meet the expectations of biological and industrial requirements.

The present study involves the synthesis of high bioactive compounds like benzimidazole, thiazole and pyrazole which exhibit various biological activities like antitumor ${ }^{10}$, analgesic ${ }^{11}$, antimicrobial ${ }^{12-13}$, antibacterial ${ }^{14-15}$ and anti-inflammatory etc.${ }^{16}$ Hence, in this direction, efforts have been undertaken to introduce most active and biologically versatile molecules containing nitrogen and sulphur triheterocyclic compounds like Benzo $[d]$ imidazole-2-yl -3, 5 substituted diphenyl -3,3a, 5, 6-Tetrahydro 2H-pyrazole thiazole derivatives which were synthesized from 2-amino-substituted benzimidazole.

\section{Materials and Methods}

\section{EXPERIMENTAL}

Progress of the reaction was observed by TLC plates. IR Spectra were recorded by Perkin Elmer BX series and ${ }^{1} \mathrm{H}$ NMR spectra were recorded by Bruker $400 \mathrm{MHz}$ instrument using DMSO as solvent and TMS as an internal standard. Chemical shifts $(\delta)$ are expressed in ppm. Mass spectra were measured on a GC/MS-QP1000EX (EI, $70 \mathrm{eV}$ ) mass spectrometer. Elemental analysis was performed on PerkinElmer $240 \mathrm{CHN}$ analyzer.

Rasayan J. Chem., 13(1), 585-592(2020)

http://dx.doi.org/10.31788/RJC.2020.1315465

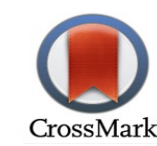


RASĀYAN J. Chem.

Vol. 13 | No. 1 |585 - 592| January - March | 2020

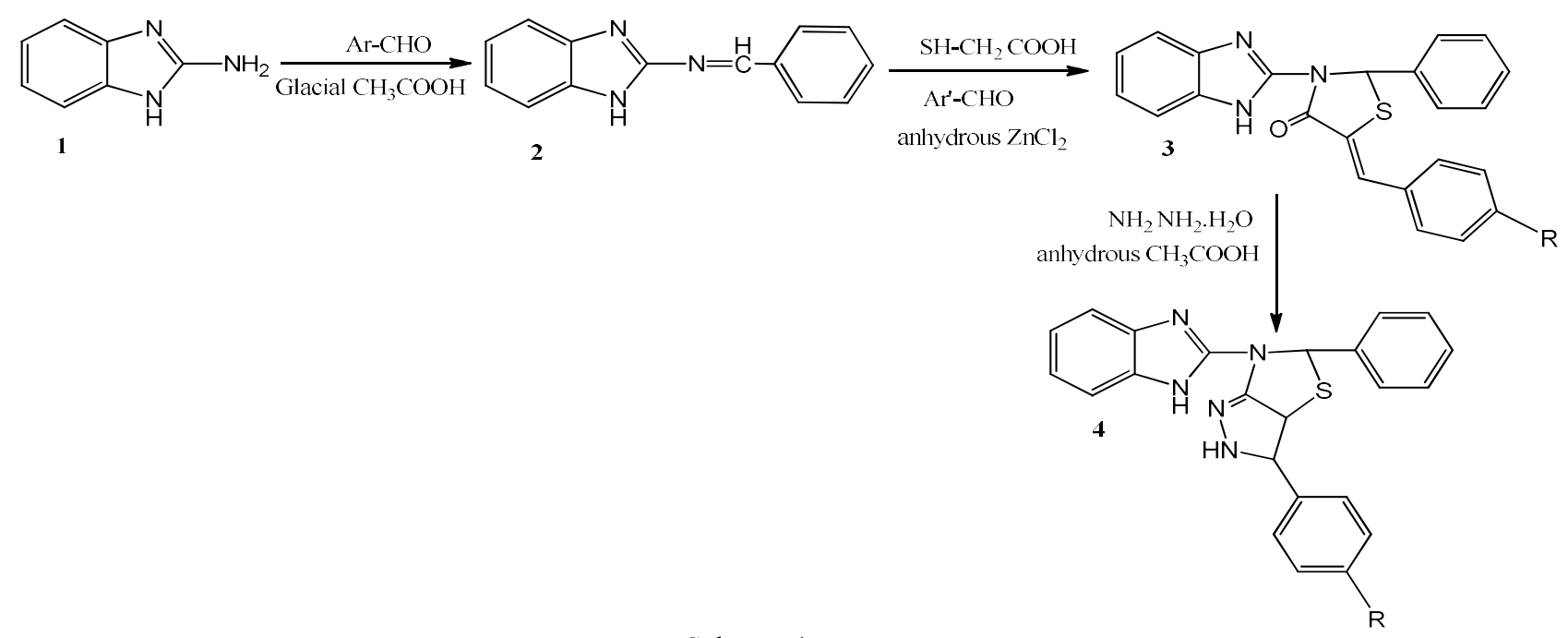

General Reaction Procedure for Compound 2

Scheme-1

A mixture of Benzaldehyde $(0.004 \mathrm{~mol})$ and 2-amino benzimidazole (1) $(0.004 \mathrm{~mol})$ with few drops of glacial acetic acid was refluxed in ethanol for about 4 hours added. Reaction progress was monitored by TLC. After the completion of the reaction, the product was cooled, filtered and dried then recrystallized with methanol to afford the compound Schiff base (2).

\section{General Reaction Procedure for Compound 3:}

An equimolar mixture of compound $2(0.01)$, mercaptoacetic acid $(0.01)$ and aromatic aldehyde $(0.01)$ in 1,4-dioxane $(30 \mathrm{ml})$ containing a small amount of $\mathrm{ZnCl}_{2}$ was refluxed for about 6 hours. The resulting product was filtered and cooled in an ice bath to attain room temperature. The solid product was filtered and washed with $10 \%$ sodium bicarbonate and recrystallized with alcohol.

The remaining compounds ( $3 \mathrm{~b}-3 \mathrm{~h}$ ) were prepared by similar procedure with minor changes as per the reaction conditions.

(Z)-3-(1H-benzo $[d]$ imidazole -2-yl )-5-benzylidene-2-phenylthiazolidin-4-one (3a)

IR $\left(\mathrm{KBr}, \mathrm{cm}^{-1}\right)$ : $3328(\mathrm{~N}-\mathrm{H}), 1548(\mathrm{C}=\mathrm{N}), 1230(\mathrm{C}=\mathrm{S}),{ }^{1} \mathrm{HNMR}\left(\mathrm{DMSO}_{-} \mathrm{d}_{6}, 400 \mathrm{MHz}, \delta\right.$ in ppm $): 5.92$ (s, 1HCH-Ar), 7.12-7.22 (m, 4H, Ar-H), 7.26-7.48 (m, 5H, Ar-H) , 7.56-7.74 ( m,5H, Ar-H), 9.85 ( br, $1 \mathrm{H}, \mathrm{NH})$. MS, m/z (\%), $383\left(\mathrm{M}^{+}\right)$; Anal. calcd for $\mathrm{C}_{23} \mathrm{H}_{17} \mathrm{~N}_{3} \mathrm{OS}: \mathrm{C}, 72.08 ; \mathrm{H}, 4.47 ; \mathrm{N}, 10.97 \%$. Found $\mathrm{C}$, $71.45 ; \mathrm{H}, 4.24 ; \mathrm{N}, 10.23 \%$.

(Z)- 3-(1H-benzo [d] imidazole -2-yl )-5-(4-methylbenzylidene)-2-phenylthiazolidin-4-one (3b) $\operatorname{IR}\left(\mathrm{KBr}, \mathrm{cm}^{-1}\right) ; 3337(\mathrm{~N}-\mathrm{H}), 2960(\mathrm{C}-\mathrm{H}), 1561(\mathrm{C}=\mathrm{N}), 1672(\mathrm{C}=\mathrm{O}), 1249(\mathrm{C}=\mathrm{S})$; ${ }^{1} \mathrm{HNMR}\left(\mathrm{DMSO}-\mathrm{d}_{6}\right.$ $, 400 \mathrm{MHz}, \delta$ in ppm): $2.85\left(\mathrm{~s}, 3 \mathrm{H}, \mathrm{CH}_{3}\right), 5.86(\mathrm{~s}, 1 \mathrm{H}, \mathrm{N}-\mathrm{CH}-\mathrm{Ar}), 7.15-7.24(\mathrm{~m}, 4 \mathrm{H}, \mathrm{Ar}-\mathrm{H}), 7.28-7.38(\mathrm{~m}$, $4 \mathrm{H}, \mathrm{Ar}-\mathrm{H})$, 7.542-7.64 ( m,4H, Ar-H), $9.96(\mathrm{br}, 1 \mathrm{H}, \mathrm{NH})$. MS , m/z (\%), $397\left(\mathrm{M}^{+}\right)$; Anal. calcd for $\mathrm{C}_{24} \mathrm{H}_{19} \mathrm{~N}_{3} \mathrm{OS}$ : C, 72.52; H, 4.82; N, $10.57 \%$. Found C, 71.81; H, 4.63; N, 10.24\%.

(Z)- 3-(1H-benzo [d] imidazole -2-yl )-5-(4-methoxybenzylidene)-2-phenylthiazolidin-4-one(3c) IR $\left(\mathrm{KBr}, \mathrm{cm}^{-1}\right) ; 3341(\mathrm{~N}-\mathrm{H}), 3046(\mathrm{C}-\mathrm{H}), 1560(\mathrm{C}=\mathrm{N}), 1678(\mathrm{C}=\mathrm{O}), 1239(\mathrm{C}=\mathrm{S}) ;{ }^{1} \mathrm{HNMR}\left(\mathrm{DMSO}-\mathrm{d}_{6}\right.$ $, 400 \mathrm{MHz}, \delta$ in ppm): $3.45\left(\mathrm{~s}, 3 \mathrm{H}, \mathrm{OCH}_{3}\right), 5.72(\mathrm{~s}, 1 \mathrm{H}, \mathrm{N}-\mathrm{CH}-\mathrm{Ar}), 7.18-7.25(\mathrm{~m}, 4 \mathrm{H}, \mathrm{Ar}-\mathrm{H}), 7.26-$ 7.51(m, 5H, Ar-H) , $7.65-7.84(\mathrm{~m}, 4 \mathrm{H}, \mathrm{Ar}-\mathrm{H}), 10.05$ ( br, 1H, NH). MS, m/z (\%), $413\left(\mathrm{M}^{+}\right)$; Anal. calcd for $\mathrm{C}_{24} \mathrm{H}_{19} \mathrm{~N}_{3} \mathrm{O}_{2} \mathrm{~S}: \mathrm{C}, 69.71 ; \mathrm{H}, 6.62 ; \mathrm{N}, 10.16 \%$. Found $\mathrm{C}, 69.02 ; \mathrm{H}, 4.36 ; \mathrm{N}, 10.02 \%$.

(Z)- 3-(1 H-benzo [d]imidazole -2-yl )-5-(4-hydroxybenzylidene)-2-phenylthiazolidin-4-one (3d) IR $\left(\mathrm{KBr}, \mathrm{cm}^{-1}\right): 3514(\mathrm{OH}), 3346(\mathrm{~N}-\mathrm{H}), 1566(\mathrm{C}=\mathrm{N}), 1235(\mathrm{C}=\mathrm{S}),{ }^{1} \mathrm{HNMR}\left(\mathrm{DMSO}-\mathrm{d}_{6}, 400 \mathrm{MHz}, \delta\right.$ in ppm): 5.88 (s, 1HCH-Ar), 7.14-7.28 (m, 4H, Ar-H), 7.30-7.48 (m, 5H, Ar-H), 7.58-7.72 ( m,5H, Ar-H), 
10.01 ( br, $1 \mathrm{H}, \mathrm{NH}), 11.28(\mathrm{~s}, 1 \mathrm{H}, \mathrm{OH}) ; \mathrm{MS}, \mathrm{m} / \mathrm{z}(\%), 428\left(\mathrm{M}^{+}\right)$; Anal. calcd for $\mathrm{C}_{23} \mathrm{H}_{16} \mathrm{~N}_{4} \mathrm{O} 3 \mathrm{~S}: \mathrm{C}, 64.47$; H, 3.76; N, $13.08 \%$. Found C, 64.23; H, 3.84; N, 12.81\%.

(Z)- 3-(1H-benzo [d]imidazole -2-yl ) -5-(4-nitrobenzylidene)-2-phenylthiazolidin-4-one (3e) IR $\left(\mathrm{KBr}, \mathrm{cm}^{-1}\right): 3340(\mathrm{~N}-\mathrm{H}), 1572(\mathrm{C}=\mathrm{N}), 1522\left(-\mathrm{NO}_{2}\right), 1684(\mathrm{C}=\mathrm{O}), 1236(\mathrm{C}=\mathrm{S}),{ }^{1} \mathrm{HNMR}\left(\mathrm{DMSO}-\mathrm{d}_{6}\right.$ ,400MHz, $\delta$ in ppm): 5.85 (s, 1HCH-Ar), 7.13-7.27 (m, 4H, Ar-H), 7.30-7.48 (m, 5H, Ar-H) , 8.12-8.34 ( m,4H, Ar-H), 10.18 ( br, $1 \mathrm{H}, \mathrm{NH}) ; \mathrm{MS}, \mathrm{m} / \mathrm{z}(\%), 399\left(\mathrm{M}^{+}\right)$; Anal. calcd for $\mathrm{C}_{23} \mathrm{H}_{17} \mathrm{~N}_{3} \mathrm{O}_{2} \mathrm{~S}: \mathrm{C}, 69.15 ; \mathrm{H}$, $4.28 ; \mathrm{N}, 10.52 \%$. Found $\mathrm{C}, 68.45 ; \mathrm{H}, 4.04 ; \mathrm{N}, 10.40 \%$.

(Z)-3-(1H-benzo[d]imidazole-2-yl)-5-(4-(dimethylamino)benzylidene)-2-phenylthiazolidin-4-one (3f) $\operatorname{IR}\left(\mathrm{KBr}, \mathrm{cm}^{-1}\right) ; 3328(\mathrm{~N}-\mathrm{H}), 1562(\mathrm{C}=\mathrm{N}), 1675(\mathrm{C}=\mathrm{O}), 1315\left(3^{0}\right.$ amine) ${ }^{1} \mathrm{HNMR}$ (DMSO-d $\mathrm{d}_{6}, 400 \mathrm{MHz}, \delta$ in ppm): $3.22\left(\mathrm{~s}, 3 \mathrm{H}, \mathrm{OCH}_{3}\right), 5.72(\mathrm{~s}, 1 \mathrm{H}, \mathrm{N}-\mathrm{CH}-\mathrm{Ar}), 7.17-7.28(\mathrm{~m}, 4 \mathrm{H}, \mathrm{Ar}-\mathrm{H}), 7.26-7.50(\mathrm{~m}, 4 \mathrm{H}, \mathrm{Ar}-\mathrm{H})$ , $7.55-7.82(\mathrm{~m}, 4 \mathrm{H}, \mathrm{Ar}-\mathrm{H}), 10.14(\mathrm{br}, 1 \mathrm{H}, \mathrm{NH}) . \mathrm{MS}, \mathrm{m} / \mathrm{z}(\%), 426\left(\mathrm{M}^{+}\right)$; Anal. calcd for $\mathrm{C}_{25} \mathrm{H}_{24} \mathrm{~N}_{4} \mathrm{OS}: \mathrm{C}$, $70.40 ; \mathrm{H}, 5.20 ; \mathrm{N}, 13.14 \%$. Found C, 69.82; H, 4.96; N, 12.62\%.

\section{(Z)-3-(1H-benzo[d]imidazol-2-yl)-5-(4-chlorobenzylidene)-2-phenylthiazolidin-4-one(3g)}

$\operatorname{IR}\left(\mathrm{KBr}, \mathrm{cm}^{-1}\right) ; 3366(\mathrm{~N}-\mathrm{H}), 1561(\mathrm{C}=\mathrm{N}), 1694(\mathrm{C}=\mathrm{O}), 875(\mathrm{C}-\mathrm{Cl}) ;{ }^{1} \mathrm{HNMR}\left(\mathrm{DMSO}-\mathrm{d}_{6}, 400 \mathrm{MHz}, \delta\right.$ in ppm): 5.84 (s, 1H, N-CH-Ar) , 7.14-7.26 (m, 4H, Ar-H), 7.25-7.48 (m, 4H, Ar-H) , 7.51 -7.82 ( m, 4H, Ar$\mathrm{H}), 10.04$ ( br, $1 \mathrm{H}, \mathrm{NH}) . \mathrm{MS}, \mathrm{m} / \mathrm{z}(\%), 433\left(\mathrm{M}^{+}\right)$; Anal. calcd for $\mathrm{C}_{23} \mathrm{H}_{16} \mathrm{ClN}_{3} \mathrm{OS}: \mathrm{C}, 66.10 ; \mathrm{H}, 3.85 ; \mathrm{N}$, $10.04 \%$. Found C, 65.82; H, 3.13; N, 9.82\%.

(Z)-5-(4-aminobenzylidene)-3-(1H-benzo[d]imidazol-2-yl)- 2-phenylthiazolidin-4-one (3h)

$\operatorname{IR}\left(\mathrm{KBr}, \mathrm{cm}^{-1}\right) ; 3365(\mathrm{~N}-\mathrm{H}), 1563(\mathrm{C}=\mathrm{N}), 1697(\mathrm{C}=\mathrm{O}) ;{ }^{1} \mathrm{HNMR}\left(\mathrm{DMSO}_{\mathrm{d}}, 400 \mathrm{MHz}, \delta\right.$ in ppm): 5.85 (s, 1H, N-CH-Ar) ,6.22( s, 2H, $\left.\mathrm{NH}_{2}\right), 7.15-7.26(\mathrm{~m}, 4 \mathrm{H}, \mathrm{Ar}-\mathrm{H}), 7.31-7.49(\mathrm{~m}, 5 \mathrm{H}, \mathrm{Ar}-\mathrm{H}), 7.58-7.82(\mathrm{~m}, 4 \mathrm{H}$, Ar-H), 10.08 ( br, $1 \mathrm{H}, \mathrm{NH})$. MS, m/z (\%), $398\left(\mathrm{M}^{+}\right)$; Anal. calcd for $\mathrm{C}_{23} \mathrm{H}_{18} \mathrm{~N}_{4} \mathrm{OS}$ : C, 69.32; H, 4.55; N, $10.04 \%$. Found C, 68.82; H, 4.13; N, 12.82\%.

\section{General Reaction Procedure for Compound 4a}

Equimolar mixture of $3 \mathrm{a}(0.03 \mathrm{~mol})$, hydrazine hydrate $(0.03 \mathrm{~mol})$ and anhydrous $\mathrm{CH}_{3} \mathrm{COONa}(0.001$ mol) in glacial acetic acid $(30 \mathrm{ml})$ were heated under reflux for about 6.5 hours, the resulting compound was cooled at room temperature and poured in to crushed ice. The product was filtered, washed with water and recrystallized with ethanol to afford the pure compound. The remaining compound (4b-4h) was prepared by similar procedure with minor changes as per the reaction conditions.

6-(1H-benzo[d]imidazole-2-yl)-3,5-diphenyl-3,3a,5,6-tetrahydro-2H-pyrazolo[3,4-d]thiazole (4a) IR (KBr, cm-1): $3348(\mathrm{~N}-\mathrm{H}), 3078(\mathrm{C}-\mathrm{H}), 1564(\mathrm{C}=\mathrm{N}), 1671(\mathrm{C}=\mathrm{O}), 1238(\mathrm{C}=\mathrm{S}), 1042(\mathrm{~N}-\mathrm{N}) ;{ }^{1} \mathrm{HNMR}$ (DMSO-d ${ }_{6}, 400 \mathrm{MHz}, \delta$ in ppm) :4.42(d,1H, CH-S), 4.85 (d, 1H, CH-N), 5.90( s,1H, N-CH-Ar), 7.107.22 ( m,4H,Ar-H), 7.26-7.45 (m,5H, Ar-H), 7.55-7.78 (m,5H,Ar-H), 9.75 (br, 1H,NH); MS, m/z (\%) 397 $\left(\mathrm{M}^{+}\right)$; Anal. Calcd for $\mathrm{C}_{23} \mathrm{H}_{19} \mathrm{~N}_{5} \mathrm{~S}: \mathrm{C}, 69.92 ; \mathrm{H}, 4.87 ; \mathrm{N}, 17.97 \%$. Found: C, 69.50; H, 4.52; N, 17.62\%.

\section{6-(1H-benzo[d]imidazole-2-yl)-5-phenyl-3-(p-tolyl)-3,3a,5,6-tetrahydro-2H-pyrazolo[3,4-d]thiazole} (4b)

IR (KBr, cm-1): $3342(\mathrm{~N}-\mathrm{H}), 3085(\mathrm{C}-\mathrm{H}), 1567(\mathrm{C}=\mathrm{N}), 1675(\mathrm{C}=\mathrm{O}), 1245(\mathrm{C}=\mathrm{S}), 1048(\mathrm{~N}-\mathrm{N}) ;{ }^{1} \mathrm{HNMR}$ (DMSO-d $\mathrm{d}_{6}, 400 \mathrm{MHz}, \delta$ in ppm) :2.85 (s, 3H, $\left.\mathrm{CH}_{3}\right), 4.60$ (d, 1H, CH-S), 4.83 (d,1H, N-CH-Ar), 7.18-7.26 (m,4H,Ar-H) ,7.28-7.38 (m,4H,Ar-H),7.45-7.68(m,4H,Ar-H), 9.95 (br, 1H,NH); MS, m/z (\%) $411\left(\mathrm{M}^{+}\right)$; Anal. Calcd for $\mathrm{C}_{24} \mathrm{H}_{21} \mathrm{~N}_{5} \mathrm{~S}$ : C, 70.15; H, 5.17; N, 17.07\%. Found: C, 69.81; H, 4.92; N, 16.82\%.

6-(1H-benzo[d]imidazole-2-yl)-3-(4-methoxyphenyl)-5-phenyl-3,3a,5,6-tetrahydro-2H-pyrazolo [3,4d] thiazole (4c)

IR (KBr, cm-1): $3343(\mathrm{~N}-\mathrm{H}), 3038(\mathrm{C}-\mathrm{H}), 1560(\mathrm{C}=\mathrm{N}), 1685(\mathrm{C}=\mathrm{O}), 1241(\mathrm{C}=\mathrm{S}), 1062(\mathrm{~N}-\mathrm{N}) ;{ }^{1} \mathrm{HNMR}$ $\left(\right.$ DMSO-d $_{6}, 400 \mathrm{MHz}, \delta$ in ppm): $3.48\left(\mathrm{~s}, 3 \mathrm{H}, \mathrm{OCH}_{3}\right), 4.62(\mathrm{~d}, 1 \mathrm{H}, \mathrm{CH}-\mathrm{S}), 4.83(\mathrm{~d}, 1 \mathrm{H}, \mathrm{N}-\mathrm{CH}-$ Ar), 5.74(s,1H,N-CH-Ar), 7.21-7.28 (m,4H,Ar-H) ,7.31-7.58 (m,4H,Ar-H), 7.68-7.87 (m,4H,Ar-H), 10.06 
(br, $1 \mathrm{H}, \mathrm{NH}) ; \mathrm{MS}, \mathrm{m} / \mathrm{z}(\%) 427\left(\mathrm{M}^{+}\right)$; Anal. Calcd for $\mathrm{C}_{24} \mathrm{H}_{21} \mathrm{~N}_{5}$ OS: C, 67.45; H, 4.95; N, $16.64 \%$. Found: C, 67.02; H, 4.26; N, 16.02\%.

\section{4-(6-(1H-benzo[d]imidazole-2-yl)-5-phenyl-3,3a,5,6-tetrahydro-2H-pyrazolo[3,4-d]thiazol-3-yl) phenol(4d)}

IR (KBr, cm-1): $3516(\mathrm{OH}), 3353(\mathrm{~N}-\mathrm{H}), 3038(\mathrm{C}-\mathrm{H}), 1571(\mathrm{C}=\mathrm{N}), 1685(\mathrm{C}=\mathrm{O}), 1242(\mathrm{C}=\mathrm{S}), 1042(\mathrm{~N}-$ $\mathrm{N})$; ${ }^{1} \mathrm{HNMR}$ (DMSO-d 6 , $400 \mathrm{MHz}, \delta$ in ppm): $4.62(\mathrm{~d}, 1 \mathrm{H}, \mathrm{CH}-\mathrm{S}), 4.78(\mathrm{~d}, 1 \mathrm{H}, \mathrm{N}-\mathrm{CH}-\mathrm{Ar}), 5.94(\mathrm{~s}, 1 \mathrm{H}, \mathrm{N}-$ CH-Ar), 7.18-7.3 (m,4H,Ar-H) ,7.28-7.54 (m,5H,Ar-H), 7.61-7.74 (m,4H,Ar-H), 10.16 (br, 1H,NH), $11.35(\mathrm{~s}, 1 \mathrm{H}, \mathrm{OH}) ; \mathrm{MS}, \mathrm{m} / \mathrm{z}(\%) 413\left(\mathrm{M}^{+}\right)$; Anal. Calcd for $\mathrm{C}_{24} \mathrm{H}_{19} \mathrm{~N}_{5} \mathrm{OS}: \mathrm{C}, 66.81 ; \mathrm{H}, 4.66 ; \mathrm{N}, 16.88 \%$. Found: C, 67.23; H, 3.96; N, 16.42\%.

\section{6-(1H-benzo[d]imidazol-2-yl)-3-(4-nitrophenyl)-5-phenyl-3,3a,5,6-tetrahydro-2H-pyrazolo[3,4-d] thiazole (4e)}

IR (KBr, cm-1): $3346(\mathrm{~N}-\mathrm{H}), 1574(\mathrm{C}=\mathrm{N}), 1528\left(\mathrm{NO}_{2}\right), 1682(\mathrm{C}=\mathrm{O}), 1235(\mathrm{C}=\mathrm{S}), 1052(\mathrm{~N}-\mathrm{N}) ;{ }^{1} \mathrm{HNMR}$ (DMSO-d 6 , 400MHz, $\delta$ in ppm): 4.75 (d, 1H, CH-S), 4.92 ( d,1H, N-CH-Ar), 5.94 (s,1H,N-CH-Ar), 7.167.30 (m,4H,Ar-H) ,7.28-7.52 (m,5H,Ar-H),8.18-8.41 (m,4H,Ar-H), 10.22 (br, 1H,NH); MS, m/z (\%) 442 $\left(\mathrm{M}^{+}\right)$; Anal. Calcd for $\mathrm{C}_{23} \mathrm{H}_{18} \mathrm{~N}_{6} \mathrm{O}_{2} \mathrm{~S}: \mathrm{C}, 62.45 ; \mathrm{H}, 4.19 ; \mathrm{N}, 18.52 \%$. Found: C, 68.45; H, 4.03; N, 10. $42 \%$.

4-(1H-benzo[d]imidazol-2-yl)-5-phenyl-3, 3a, 5, 6-tetrahydro-2H-pyrazolo [3,4-d] thiazole -3-yl)$\mathrm{N}, \mathrm{N}$ dimethyl amine (4f)

IR (KBr, cm-1): $3334(\mathrm{~N}-\mathrm{H}), 1564(\mathrm{C}=\mathrm{N}), 1678(\mathrm{C}=\mathrm{O}), 1235(\mathrm{C}=\mathrm{S}), 1323$ ( $3^{0}$ amine $), 1052(\mathrm{~N}-\mathrm{N})$; ${ }^{1} \mathrm{HNMR}$ (DMSO-d $\mathrm{d}_{6}, 400 \mathrm{MHz}, \delta$ in ppm): 3.27(s,6H-N $\left.\left(\mathrm{CH}_{3}\right)_{2}\right), 4.75(\mathrm{~d}, 1 \mathrm{H}, \mathrm{CH}-\mathrm{S}), 4.91(\mathrm{~d}, 1 \mathrm{H}, \mathrm{N}-\mathrm{CH}-$ Ar), 5.74(s,1H,N-CH-Ar), 7.21-7.30 (m,4H,Ar-H) ,7.36-7.55 (m,4H,Ar-H), 7.62-7.84 (m,4H,Ar-H), 10.18 (br, $1 \mathrm{H}, \mathrm{NH}) ; \mathrm{MS}, \mathrm{m} / \mathrm{z}(\%) 440\left(\mathrm{M}^{+}\right)$; Anal. Calcd for $\mathrm{C}_{25} \mathrm{H}_{24} \mathrm{~N}_{6} \mathrm{~S}$ : C, 68.16; H, 5.48; N, $19.04 \%$. Found: C, 68.86; H, 4.93; N, 18. 62\%.

6-(1H-benzo[d]imidazol-2-yl)-3-(4-chlorophenyl)-5-phenyl-3, 3a, 5, 6-tetrahydro-2H-pyrazolo [3, 4d] thiazole (4g)

IR (KBr, cm-1): $3382(\mathrm{~N}-\mathrm{H}), 1565(\mathrm{C}=\mathrm{N}), 1698(\mathrm{C}=\mathrm{O}), 1235(\mathrm{C}=\mathrm{S}), 872(\mathrm{C}-\mathrm{Cl}), 1052(\mathrm{~N}-\mathrm{N})$; ${ }^{1} \mathrm{HNMR}$ (DMSO-d 6 , 400MHz, $\delta$ in ppm): 4.73 (d, 1H, CH-S), 4.91 ( d,1H, N-CH-Ar), 5.84 (s,1H,N-CH-Ar), 7.187.32 (m,4H,Ar-H) ,7.36-7.52 (m,4H,Ar-H),7.60-7.85 (m,4H,Ar-H), 10.16 (br, 1H,NH); MS, m/z (\%) 431 $\left(\mathrm{M}^{+}\right)$; Anal. Calcd for $\mathrm{C}_{23} \mathrm{H}_{26} \mathrm{~N}_{5} \mathrm{~S}: \mathrm{C}, 66.96 ; \mathrm{H}, 4.26 ; \mathrm{N}, 16.25 \%$. Found: C, 66.24; H, 4.03; N, 15.82\%.

\section{4-(6-(1H-benzo[d]imidazol-2-yl)-5-phenyl-3,3a,5,6-tetrahydro-2H-pyrazolo[3,4-d]thiazol-3-yl)} aniline (4h)

IR (KBr, cm-1): $3375(\mathrm{~N}-\mathrm{H}), 1565(\mathrm{C}=\mathrm{N}), 1695(\mathrm{C}=\mathrm{O}), 1232(\mathrm{C}=\mathrm{S}), 1052(\mathrm{~N}-\mathrm{N}) ;{ }^{1} \mathrm{HNMR}\left(\mathrm{DMSO}-\mathrm{d}_{6}\right.$ , $400 \mathrm{MHz}, \delta$ in ppm): $4.75(\mathrm{~d}, 1 \mathrm{H}, \mathrm{CH}-\mathrm{S}), 4.94(\mathrm{~d}, 1 \mathrm{H}, \mathrm{N}-\mathrm{CH}-\mathrm{Ar}), 5.94(\mathrm{~s}, 1 \mathrm{H}, \mathrm{N}-\mathrm{CH}-\mathrm{Ar}), 6.22(\mathrm{~s}, 2 \mathrm{H}$, NH2), 7.18-7.32 (m,4H,Ar-H) , 7.38-7.55 (m,4H,Ar-H), 7.62-7.92 (m,4H,Ar-H), 10.06 (br, 1H,NH) ; MS, $\mathrm{m} / \mathrm{z}(\%) 398\left(\mathrm{M}^{+}\right)$; Anal. Calcd for $\mathrm{C}_{23} \mathrm{H}_{20} \mathrm{~N}_{6} \mathrm{~S}: \mathrm{C}, 66.97 ; \mathrm{H}, 4.86 ; \mathrm{N}, 20.36 \%$. Found: $\mathrm{C}, 66.64 ; \mathrm{H}, 4.43$; $\mathrm{N}, 20.04 \%$.

\section{RESULTS AND DISCUSSION}

The present study reports the synthesis of thiazolo-pyrazole derivatives linked with benzimidazole with an appreciable yield. The compound $\mathbf{2}$ (Schiff base) is synthesized using 2-aminobenzimidazole with Benzaldehyde under simple conditions. The compound $\mathbf{3}$ ( Chalcone derivatives of thiazolidinone) is synthesized by one-pot three-component cyclization using compound $\mathbf{2}$, mercaptoacetic acid and aromatic aldehyde using anhydrous $\mathrm{ZnCl}_{2}$. Later, the Chalcone derivative of thiazolidinone (3) undergoes cyclization with hydrazine hydrate in the presence of anhydrous acetic acid to afford the compound $\mathbf{4}$. The structures of synthesized compounds were established based on spectral and analytical data. The compounds showed IR absorption bands at $3366 \mathrm{~cm}^{-1}(\mathrm{NH}), 1568 \mathrm{~cm}^{-1}(\mathrm{C}=\mathrm{N}), 1241 \mathrm{~cm}^{-1}(\mathrm{C}=\mathrm{S}), 1680 \mathrm{~cm}^{-}$ ${ }^{1},(\mathrm{C}=\mathrm{O})$ respectively. ${ }^{1} \mathrm{HNMR}$ spectra of test compounds displayed singlet signals at 5.58 for N-CH-Ar, doublet signals at $4.62 \mathrm{CH}-\mathrm{S}, 4.82 \mathrm{CH}-\mathrm{N}$, and $9.82 \mathrm{ppm}$ for $\mathrm{NH}$ protons and also phenylic protons as 
RASĀYAN J. Chem.

Vol. 13 | No. 1 |585 - 592| January - March | 2020

multiplet in the range of 7.10-8.10 ppm. Mass spectra of synthesized compounds showed a molecular ion peak at $\mathrm{m} / \mathrm{z}$ concerning their molecular weights.

\begin{tabular}{|c|c|c|c|c|c|c|c|c|}
\hline \multicolumn{9}{|c|}{ Table-1: Physical Data of Synthesized Compounds (3a-3h) and (4a-4h) } \\
\hline Compounds & $3 a$ & $3 b$ & $3 \mathrm{c}$ & $3 d$ & $3 \mathrm{e}$ & $3 \mathrm{f}$ & $3 g$ & $3 \mathrm{~h}$ \\
\hline $\mathrm{R}$ & $\mathrm{Ar}$ & 4- $\mathrm{CH}_{3}-\mathrm{Ar}$ & $4-\mathrm{OCH}_{3}-\mathrm{Ar}$ & 4-OH- Ar & 4- $\mathrm{NO}_{2}-\mathrm{Ar}$ & 4- $\mathrm{N}\left(\mathrm{CH}_{3}\right)_{2}-\mathrm{Ar}$ & 4-Cl-Ar & 4- $\mathrm{NH}_{2}-\mathrm{Ar}$ \\
\hline M.P $\left({ }^{\circ} \mathrm{C}\right)$ & $208-12$ & $215-17$ & $220-22$ & $213-14$ & $221-23$ & $225-28$ & $215-17$ & $210-12$ \\
\hline Yield (\%) & 80 & 82 & 78 & 81 & 76 & 75 & 76 & 80 \\
\hline Compounds & $4 \mathrm{a}$ & $4 \mathrm{~b}$ & $4 \mathrm{c}$ & $4 \mathrm{~d}$ & $4 \mathrm{e}$ & $4 \mathrm{f}$ & $4 \mathrm{~g}$ & $4 \mathrm{~h}$ \\
\hline $\mathrm{R}$ & $\mathrm{Ar}$ & $4-\mathrm{CH}_{3}-\mathrm{Ar}$ & $4-\mathrm{OCH}_{3}-\mathrm{Ar}$ & $4-\mathrm{OH}-\mathrm{Ar}$ & $4-\mathrm{NO}_{2}-\mathrm{Ar}$ & 4- $\mathrm{N}\left(\mathrm{CH}_{3}\right)_{2}-\mathrm{Ar}$ & 4-Cl-Ar & $4-\mathrm{NH}_{2}-\mathrm{Ar}$ \\
\hline $\mathrm{M} . \mathrm{P}\left({ }^{\circ} \mathrm{C}\right)$ & $238-40$ & $245-47$ & $250-52$ & $243-46$ & $250-52$ & $255-57$ & $246-48$ & $241-42$ \\
\hline Yield (\%) & 82 & 80 & 75 & 72 & 74 & 76 & 73 & 72 \\
\hline
\end{tabular}

In response to nitrate, Mycobacterium which causes tuberculosis produces nitrate reductase by NarL. ${ }^{17-19}$ NarL is a cytoplasmic response regulator that is involved in regulating gene expression of this bacterium. NarL has been identified as a potential drug target due to its role in nitrate respiration. ${ }^{20}$ The Docking studies were conducted on NarL similar to that reported by Prashantha et al ${ }^{21}$ using docking server software. ${ }^{22-23}$ The protein structure was downloaded from the Protein data bank and was docked to the ligand. The estimated free energy of binding was found to be $-7.16 \mathrm{kcal} / \mathrm{mol}$ while the inhibition constant was found to be $5.60 \mathrm{uM}$ and ARG63 was involved in polar bond formation with the ligand (Tables-2, 3 and 4). The docking pose of the ligand with NarL is shown in Fig.-1.

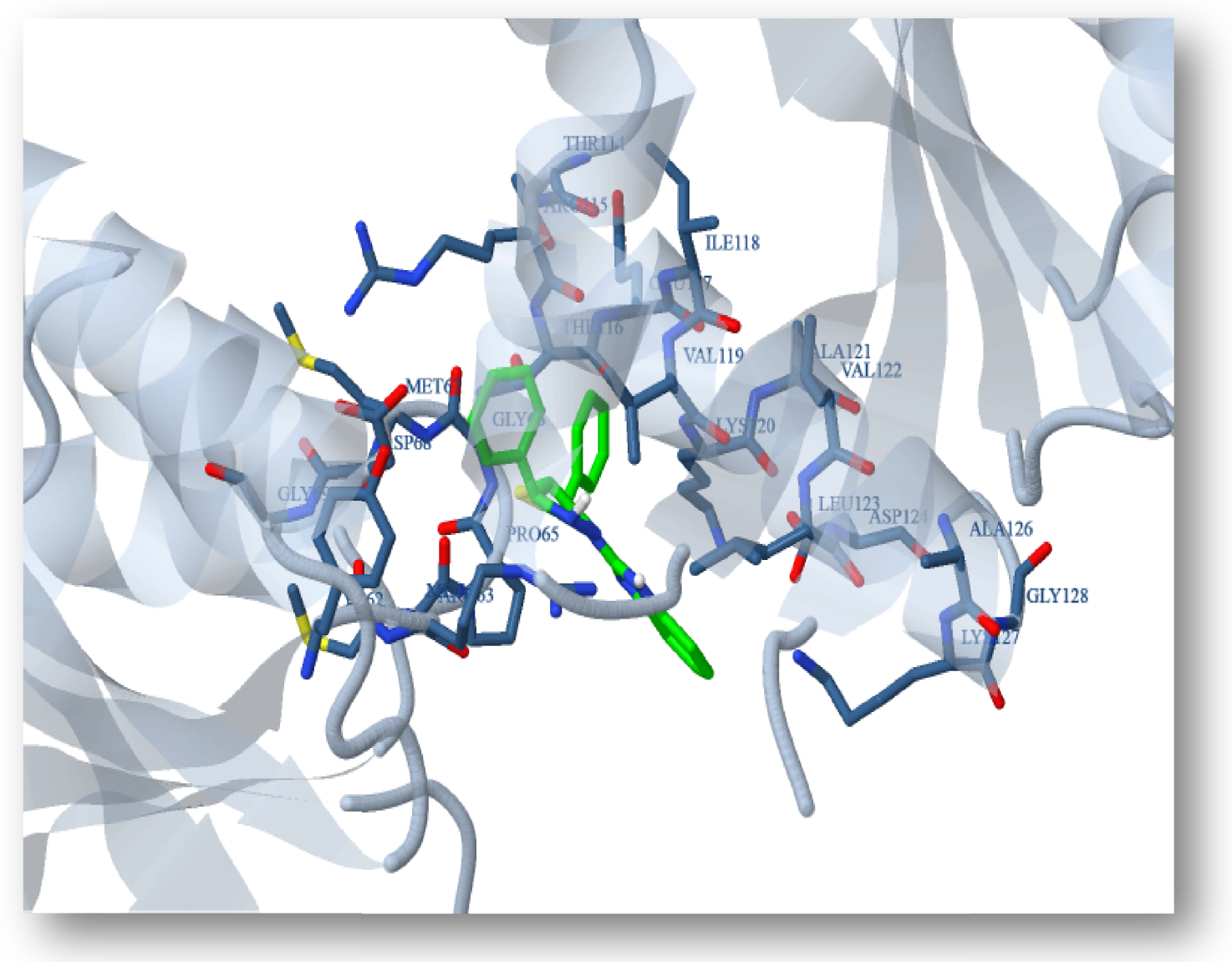

Fig.-1: Docking Pose between the Ligand and the Protein 589 
RASĀYAN J. Chem.

Vol. 13 | No. 1 |585 - 592| January - March | 2020

Table-2: Free Energy of Binding between the Ligand and the Protein

\begin{tabular}{c|c|c|c|c|c|c}
\hline $\begin{array}{c}\text { Estt. Free } \\
\text { Energy Binding }\end{array}$ & $\begin{array}{c}\text { Estt. } \\
\text { Inhibition } \\
\text { Constant, Ki }\end{array}$ & $\begin{array}{c}\text { vdW + H } \\
\text { Bond + Desolv. } \\
\text { Energy }\end{array}$ & $\begin{array}{c}\text { Electrostatic } \\
\text { Energy }\end{array}$ & $\begin{array}{c}\text { Total } \\
\text { Intermolecular. } \\
\text { Energy }\end{array}$ & Frequency & $\begin{array}{c}\text { Interact } \\
\text { Surface }\end{array}$ \\
\hline$-7.16 \mathrm{kcal} / \mathrm{mol}$ & $5.60 \mathrm{uM}$ & $-7.73 \mathrm{kcal} / \mathrm{mol}$ & $\begin{array}{c}-0.07 \\
\mathrm{kcal} / \mathrm{mol}\end{array}$ & $-7.79 \mathrm{kcal} / \mathrm{mol}$ & $50 \%$ & 809.37 \\
\hline
\end{tabular}

Table-3: Decomposed Interaction Energies in Kcal/mole

\begin{tabular}{c|c|c}
\hline Polar & Hydrophobic & Other \\
\hline ARG63 (0) & PRO65 $(0)$ & ARG115 (0) \\
\hline & VAL119 $(0)$ & ASP68 (0) \\
\hline & & GLU117(0) \\
\hline & & LYS120 (0) \\
\hline
\end{tabular}

Table-4: Interaction Showing the Various Bonds between the Ligand and the Protein

\begin{tabular}{|c|c|c|c|c|}
\hline \multicolumn{2}{|c|}{ Polar } & Hydrophobic & \multicolumn{2}{|c|}{ Other } \\
\hline $\begin{array}{r}\text { N4 }(15) \\
\quad[3.06]\end{array}$ & $\begin{array}{c}\text { ARG63 } \\
\text { (CZ, } \\
\text { NE, } \\
\text { NH2) } \\
\end{array}$ & $\begin{array}{cc}\text { C17 (23) } & \text { VAL119 } \\
{[3.01]} & -\quad(\mathrm{CB}, \\
& \text { CG2) }\end{array}$ & $\begin{array}{c}\text { C16 (22) } \\
\quad[3.38]\end{array}$ & $\begin{array}{c}\text { ARG11 } \\
-\quad 5(\mathrm{CG} \\
\mathrm{NE})\end{array}$ \\
\hline $\begin{array}{r}\text { N5 (16) } \\
{[2.66]}\end{array}$ & $\begin{array}{c}\text { ARG63 } \\
\text { (CZ, } \\
-\quad \mathrm{NE}, \\
\mathrm{NH} 2) \\
\end{array}$ & $\begin{array}{cc}\text { C18 (24) } & \text { VAL119 } \\
{[3.19]} & \text { (CB, } \\
& \text { CG1, } \\
& \text { CG2) } \\
\end{array}$ & $\begin{array}{l}\text { C17 } \\
(23) \\
{[3.48]}\end{array}$ & $\begin{array}{r}\mathrm{ARG} 11 \\
5(\mathrm{CG})\end{array}$ \\
\hline \multirow[t]{9}{*}{$\begin{array}{r}\mathrm{H} 8(37) \\
{[2.74]}\end{array}$} & $\begin{array}{c}\text { ARG63 } \\
\text { (CZ, } \\
\text { NE, } \\
\text { NH2) } \\
\end{array}$ & $\begin{array}{c}\text { C16 (22) } \\
{[3.85]}\end{array}-\begin{array}{c}\text { VAL119 } \\
\text { (CG2) }\end{array}$ & $\begin{array}{c}\text { C9 (12) } \\
\quad[3.24]\end{array}$ & $\begin{array}{c}\text { THR116 } \\
-\quad(\mathrm{CB}, \\
\text { CG2) }\end{array}$ \\
\hline & & $\begin{array}{c}\text { C10 (14) } \\
{[3.40]}\end{array}-\begin{array}{c}\text { PRO65 } \\
(\mathrm{CB})\end{array}$ & $\begin{array}{l}\mathrm{S} 1(13) \\
\quad[3.16]\end{array}$ & $\begin{array}{c}\text { THR116 } \\
-\quad(\mathrm{CB}, \\
\text { CG2) }\end{array}$ \\
\hline & & & $\begin{array}{c}\mathrm{C} 19(25) \\
{[2.93]}\end{array}$ & $\begin{array}{c}\text { THR116 } \\
-\quad(\mathrm{CB}, \\
\text { OG1) }\end{array}$ \\
\hline & & & $\begin{array}{c}\mathrm{C} 20(26) \\
\quad[3.13]\end{array}$ & $\begin{array}{c}\text { THR116 } \\
-\quad(\mathrm{CB}, \\
\text { OG1) } \\
\end{array}$ \\
\hline & & & $\begin{array}{c}\mathrm{C} 18(24) \\
{[3.33]}\end{array}$ & $\begin{array}{c}\text { THR116 } \\
-\quad(\mathrm{CB}, \\
\text { CG2) } \\
\end{array}$ \\
\hline & & & $\begin{array}{c}\mathrm{C} 13(19) \\
{[3.52]}\end{array}$ & $\begin{array}{c}\text { THR116 } \\
\text { (CG2) }\end{array}$ \\
\hline & & & $\begin{array}{c}\mathrm{C} 17 \text { (23) } \\
{[3.75]}\end{array}$ & $\begin{array}{c}\text { THR116 } \\
\text { (CG2) }\end{array}$ \\
\hline & & & $\begin{array}{c}\text { C20 (26) } \\
{[3.74]}\end{array}$ & $\begin{array}{c}\text { GLU117 } \\
(\mathrm{CG})\end{array}$ \\
\hline & & & $\begin{array}{r}\text { H8 }(37) \\
{[2.15]}\end{array}$ & $\begin{array}{c}\text { VAL119 } \\
\text { (CB, } \\
\text { CG1, } \\
\text { CG2) }\end{array}$ \\
\hline
\end{tabular}


RASĀYAN J. Chem.

Vol. 13 | No. 1 |585 - 592| January - March | 2020

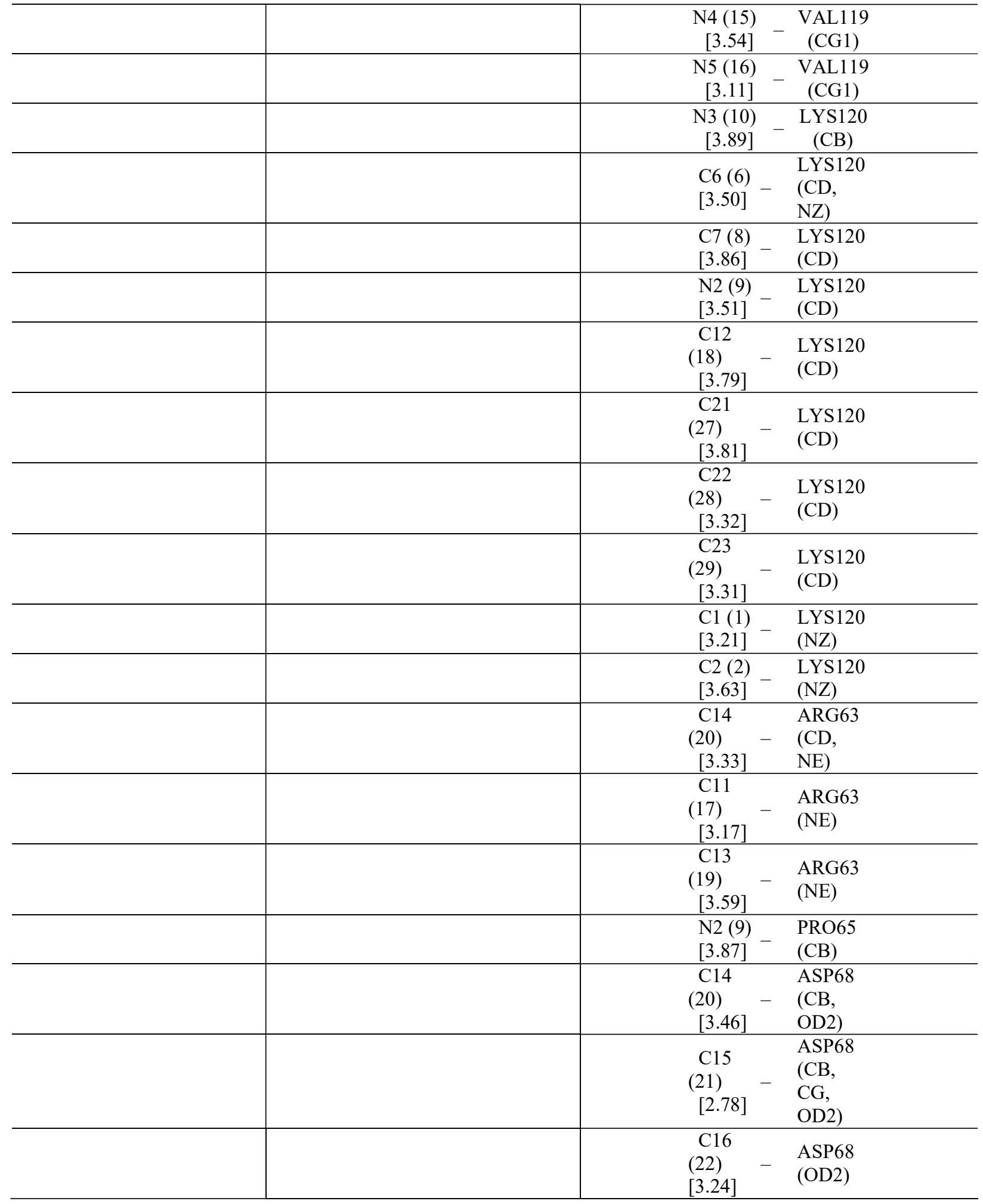

CONCLUSION

The experimental results conclude that a new series of thiazolo -pyrazole containing benzimidazole nucleus and its derivatives are successfully synthesized. It has been reported in the present work that the title compounds (i.e., tri heterocyclic compounds) are exhibiting antitubercular activity based on In silico studies. 
RASĀYAN J. Chem.

Vol. 13 | No. 1 |585 - 592| January - March | 2020

\section{ACKNOWLEDGMENT}

The authors are thankful to the Department of Chemistry, Kakatiya University for its constant support during the work and also thankful to the Department of Bio-Chemistry, Mahatma Gandhi UniversityNalgonda, India.

\section{REFERENCES}

1. A. C. Valery, V. Fokin, Chemical Reviews, 109(2), 725 (2009), DOI: 10.1021/cr 800448q

2. B.Jiang, T.Rajale, W.Wever, S.Jiang, Chemistry An Asian Journal, 5 (11), 2318(2010), DOI: 10.1002/asia.201000310.

3. S.Hassan, Thamson. J. J. Müller, Advanced Synthesis \& Catalysis, 357(4), 617(2015), DOI: $10.1002 /$ adsc. 201400904.

4. A.F. Sherif Rostam, I.M. EI -Ashmawy, A.Heba.Abd EI Razik, Mona H. Badr, M.A. Hayam Ashour Bioorganic and Medicinal Chemistry., 17 (2), 882(2009), DOI: 10:1016/ jbmc 2008. 11.035.

5. Y. Shiraishi, Y.Sugano, S.Tanaka, Angew. Chemie international Edition, 49(9), 1656 (2010), DOI: 10.1002/anie.200906573.

6. A. Ziarati , A. Badiei , G. M. Ziarani , H. Eskandarloo, Catalysis Communications, 95, 77(2017), DOI: $10.1016 /$ jcatcom.2017.02.023.

7. K.Gullapelli, G. Brahmeshwari, M. Ravichander, Uma Kusuma, Egyptian Journal of Basic and Applied Sciences, 4 (4), 303 (2017), DOI: 10.1016/j.ejbas.2017.09.002.

8. N C,Desai , Amit M.Dodiya, Niraj R.Shihory, Medicinal Chemistry Research, 21 (9), 2579 (2012), DOI: $10.1007 /$ S00044-011-9782-2.

9. K.Ramalingam, D.Ramesh, B.Sreenivasulu, Rasayan Journal of Chemistry, 12(2), 583(2019), DOI: 10.31788/RJC.2019.1225096

10. S. Ahmed Galal, A. S. Abdelsamie, M. L. Rodriguez, S. M. Kerwin, H. I.El Diwani., European Journal of Chemistry, 1(2), 67(2010), DOI:10.5155/eurjchem.1.2.67-72.1.

11. S. Dixit P. Kumar Sharma N. Kaushik, Medicinal Chemistry Research, 22(2), 900(2013), DOI: 10.1007/s00044-012-0083-1.

12. D.S. Ngono Bikob, D.C.Vodnar,A. Stana, B. Tiperciuc, C. Nastasa, M. Douchet, O. Oniga, Journal of Saudi Chemical Society, 21(7), 861(2017), DOI:10.1016/j.jscs.2017.04.007

13. M.Idrees, S.Kola, N.J. Siddiqui , Rasayan Journal of Chemistry, 12(4), 1733(2019), DOI: 10.31788/rjc.2019.1245467.

14. L.GangYu, T. FengNi, W.Gao, YuanHe, Y.Y.Wang, H.Weicui, C.G.Yang, W.WeiQiu ., European Journal Medicinal Chemistry,90(27),10(2014),DOI:10.1016/j.ejmech.2014.11.015.

15. K.Pradeep, G. Shashikant, L.Tukaram, SK Abdul, S.Babasaheb, P.Choudhari, B.Madhusudhan, Rasayan Journal of Chemistry, 11(14), 1441 (2018), DOI: 10.31/RJC.2018.1143080.

16. M.Shivanand, P.Vijaykumar, V.Ravikumar, Rasayan Journal of Chemistry, 12(4), 1810(2019), DOI: 10.31788/RJC..2019.1245375.

17. R. Cavicchioli, I. Schroder, M. Constanti, and R. P. Gunsalus, Journal of Bacteriology, 177(9), 2416(1995), DOI:10.1128/jb.177.9.2416-2424.1995.

18. H. Wang, R.P. Gunsalus, Journal of Bacteriology, 185 (17), 5076(2003), DOI: 10.1128/ JB. 185. 17. 5076-5085.2003.

19. I. Schroder, C. D. Wolin, R. Cavicchioli, and R. P. Gunsalus, Journal of Bacteriology, 176(16), 4985 (1994), DOI:10.1128/jb.176.16.4985-4992.1994.

20. J. H. Zhang, G. Xiao, R. P. Gunsalus, and W. L. Hubbell, Biochemistry, 42 (9), 2552 (2003), DOI: $10.1021 / \mathrm{bi0272205.}$

21. P. Karunakar, C.R. Girija, V. Krishnamurthy, V. Krishna, and K. V. Shivakumar, Tuberculosis Research and Treatment, 697532, 10 (2014), DOI:10.1155/2014/697532.

22. Z.Bikadi, L.Demko, E.Hazai, Archives of Biochemistry and .Biophysics, 461(2), 225(2007), DOI: 10. 1016/j.abb.2007.02.020.

23. K. Ian, McDonald, J.M. Thornton, Journal of Molecular Biology, 238(5), 777 (1994), DOI: 10.1006 /jmbi. 1994. 1334.

[RJC-5465/2019] 